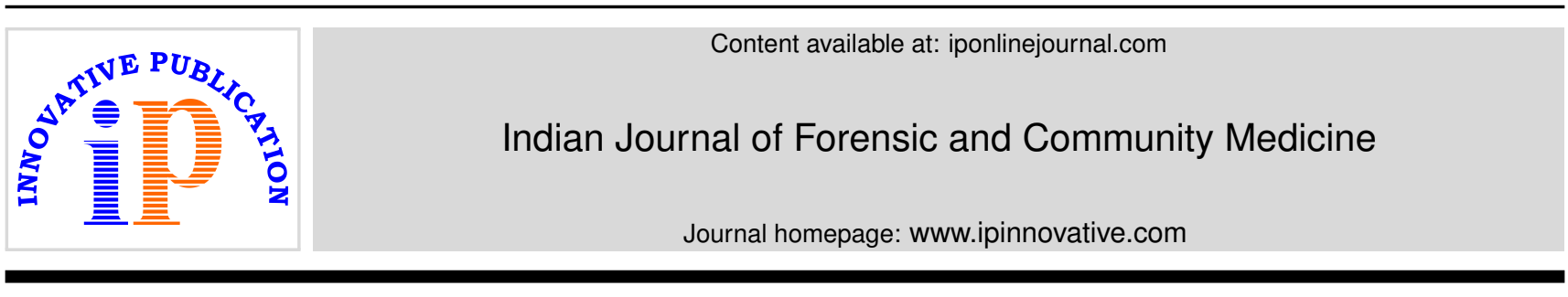

Original Research Article

\title{
A histopathological study of visceral organs in death due to burns
}

\author{
Avinash J Pujari ${ }^{1}$, Rahul V Kedare ${ }^{2, *}$ \\ ${ }^{1}$ Dept. of Forensic Medicine \& Toxicology, MIMER Medical College, Pune, Maharashtra, India \\ ${ }^{2}$ Dept. of Pharmacology, MIMER Medical College, Pune, Maharashtra, India
}

\section{A R T I C L E I N F O}

\section{Article history:}

Received 30-04-2020

Accepted 26.05.2020

Available online 31-07-2020

Keywords:

Autopsy

Burn

Histopathological changes

\begin{abstract}
A B S T R A C T
Background: Burns is a burning problem perhaps ever since human being existence particularly, from its use of fire. Now-a-days due to increasing industrialization and urbanization, we are facing this problem in greater magnitude. Earlier studies described burns as a silent epidemic. Besides causing death in some cases, it is the cause of lifelong disfigurement, deformity and disability of the body. Major burn-injury is also the cause of psychological disturbance and financial loss to the individual/ victim and also the family and society at large. Our aim is to examine histopathology in different organ and skin affected due to burn. Materials and Methods: A cross-sectional descriptive study was conducted over a period from February 1988 to November 1989. Study of cytomorphological changes in visceral organs particularly brain, heart, lung, liver, spleen, kidney and adrenal in cases of death due to burns, received at autopsy section of Forensic Medicine and Toxicology department, Government Medical College, Aurangabad, Maharashtra.

Results: In our study, burn due to domestic accidents found in $83.80 \%$. The inflammatory material that was instrumental in causing burn injury was found as kerosene in 90(69.23\%), cloths worn (flames) in 35 $(26.92 \%)$ cases, diesel in 2 cases $(01.53 \%)$, gasoline, petrol scalding in $1(0.76 \%)$ case each; we found that burn were caused by inflammable liquids in $71.20 \%$ cases. Based on history and dying declaration, nature of burn injury was assumed as accidental in $110(84.62 \%)$ cases, suicidal in $19(14.06 \%)$ cases and homicidal in $1(00.76 \%)$ cases.

Conclusion: Present study shows that most of the cases died within 7 days of sustaining burns. Microscopic examination of visceral organs such as brain, heart, lung, liver, spleen, kidney and adrenal were carried out and morphological changes were observed in each organ. Microscopic observations were recorded and analyzed keeping in view the time sequence after burn incidence. Congestion of various organs was seen in all cases which died within first 24 hours.
\end{abstract}

(C) 2020 Published by Innovative Publication. This is an open access article under the CC BY-NC license (https://creativecommons.org/licenses/by-nc/4.0/)

\section{Introduction}

Burns is a burning problem perhaps ever since human being existence particularly from its use of fire. ${ }^{1}$ Now-a-days due to increasing industrialization and urbanization, we are facing this problem in greater magnitude. Previous studies described burns as a silent epidemic. Besides causing death in some cases, it is the cause of lifelong disfigurement, deformity and disability of the body. Major burn-injury is also the cause of psychological disturbance and financial loss to the individual/ victim and also the family and society

\footnotetext{
* Corresponding author.

E-mail address: rahulkedare999@gmail.com (R. V. Kedare).
}

at large. Fire constitutes the third leading cause of death in U.S.A. Burns constitute a major cause of death and the morbidity in India. Accidental, suicidal and homicidal deaths due to burns are all common in India. ${ }^{2}$ Burns are occurring in all circumstances of human- life i.e. domestic, peridomestic, industrial, environmental etc. In India burns due to domestic accidents are most common and are also causing the most exhaustive and severe injuries to the body. Mortality due to burns is related with so many factors e.g. age, sex, severity of burns, nature of the agent causing burns, time of the burn injury, time interval between injury and treatment etc. considering the low socio- economic status 
of women in India. They are more vulnerable to sustain the burn injury. Social problems like dowry, illiteracy, ignorance etc. contribute for female preponderance in burn occurrence. Females are set on fire for financial gains, for suspicions of chastity or infidelity. Although it is difficult to decide the exact cause of death in most of cases of burns an attempt has been made by various authors to determine roles of various factors like sepsis, acute renal failure, burns shock, neurogenic shock etc in causation of death in case of burn injury. It is well known fact that all organs are affected due to burns. But most commonly the cytomorphological changes are seen in brain, heart, lungs, liver, spleen, kidney and adrenals. ${ }^{3}$

\section{Materials and Methods}

A cross-sectional descriptive study was conducted over a period from February 1988 to November 1989. Study of cytomorphological changes in visceral organs particularly brain, heart, lung, liver, spleen, kidney and adrenal in cases of death due to burns, received at autopsy section of Forensic Medicine and Toxicology department, Government Medical College, Aurangabad, Maharashtra. The study was carried out prior to 1993 i.e. in the 198889, when Institutional Ethics Committee Clearance wasn't required / mandatory. This work includes the study of 130 cases of burns admitted during the period from February 1988 to November 1989. There were no specific selection of cases but specimen were obtained from those consecutive autopsy cases of death due to burns, on whom the autopsy was performed within 6 hours of death. On admission extent of body surface affected due to burns was calculated as per rules of nine. Classification of burns was done according to Wilson as epidermal, dermo-epidermal and deep burns. The detailed clinical examination and necessary investigation of the patients were carried out according to the proforma.

All the specimens were preserved in $10 \%$ formalin for fixation. In histo-pathological examination, number of sections were taken from different sites according to size of specimen. Then these sections were processed. After processing the section were embedded in paraffin, cut with microtome at $5 \mathrm{~mm}$ thickness and stained with Hematoxylin and Eosin (H\&E). Special staining procedure like Periodic Acid Schiff (PAS) was done as and when required.

All relevant data recorded, were analyzed by using Statistical Package for the Social Sciences (SPSS) software and the results were calculated in the form of frequency and percentage.

\section{Observations}

There were total 130 cases, out of which 35 were male and 95 were female.

In this study the minimum age was 1 year and maximum was 75 years. Religion wise distribution of the subject is as follows.

The Table 1 and 2 showed that 15 cases were unmarried and rest 115 were married $(11.54 \%$ and $88.46 \%$ respectively). Maximum cases were persons between age group of 21 to $40 \mathrm{yrs}^{4}{ }^{4}(77$ out of 130 - i.e. $59.24 \%)$.

According to the nature of burn injury (as per history given by patient or dying declaration). Survival period of maximum cases was between 1 to 7 days, 70 out of 130 (i.e. $53.85 \%$.)

Distribution of cases according to predominant factor as to the cause of death in Table 4 .

\section{Discussion}

Out of 130 cases $15(11.54 \%)$ were unmarried and rest $115(88.46 \%)$ were married. Majority of women were recently married and there is room to believe that burn incidents may be related to dowry related problems. The majority i.e. 105 out 130 was Hindus, 25 were Muslim and 2 being christen, represented the demographic distribution of population. Manner of sustaining the burns was studied and it was found that burns were due to bursting of stoves in 52(40\%) cases, soaking clothes with kerosene in $18(13.84 \%)$, fall of chimany (Kerosene lamp) on body. Saree catching fire from while warming body in $9(06.92 \%)$ cases, bursting of diesel tank in $2(01.53 \%)$ cases, due to boiling water causing burns, pouring petrol on body (self), leakage of cooking gas causing fire, petromax bursting in $1(00.76 \%)$ each. These findings are comparable to observations recorded by Forester and Richardson. ${ }^{5}$ Domestic burns due to cooking accidents were found in $62.29 \%$ of cases in our study and these are similar to the findings noted by Joanne G. parks et al. ${ }^{6}$ in their study as $57 \%$ cases. This is due to fact that the females are busy to prepare the food in home along with various other works. In our study, burn due to domestic accidents was found in $83.80 \%$. The inflammatory material that was instrumental in causing burn injury was found as kerosene in 90(69.23\%), cloths worn (flames) in 35 (26.92\%) cases, diesel in 2 cases $(01.53 \%)$, gasoline, petrol scalding in $1(00.76 \%)$ case each; we found that burn were caused by inflammable liquids in $71.20 \%$ cases whereas Forester and Richardson found in $18.60 \%$ cases.

Based on history and dying declaration, nature of burn injury was assumed as accidental in $110(84.62 \%)$ cases, suicidal in $19(14.06 \%)$ cases and homicidal in $1(00.76 \%)$ cases (Table 2). Bull had observed similar findings in accidental cases. $^{7}$

Congestion of brain was observed in all the cases which succumbed to injury within 24 hours of sustaining burns. Petechial haemorrhages seen in few cases can be attributed to anoxia. Oedema was seen in few cases belonging to each group of survival period. Jackson et al had noted liquefaction necrosis of brain in their studies. No such necrosis was found in this study except degenerative 
Table 1: Religion wise distribution of the subject

\begin{tabular}{lcccc}
\hline Religion & Male & Female & Total & Percentage $(\%)$ \\
Hindu & 28 & 77 & 105 & 80.76 \\
Muslim & 06 & 17 & 23 & 17.60 \\
Christian & 01 & 01 & 02 & 01.53 \\
Total & 35 & 95 & 130 & 100 \\
\hline
\end{tabular}

Table 2: Types of burn injury

$\begin{array}{lcc}\text { Nature of burn Injury } & \text { No. of Cases } & \text { Percentage }(\%) \\ \text { Accidental } & 110 & 84.62 \\ \text { Suicidal } & 19 & 14.62 \\ \text { Homicidal } & 01 & 00.76 \\ \text { Total } & 130 & 100\end{array}$

Table 3: Percentage of body surface involved in burn injury

\begin{tabular}{lcc}
\hline Percentage of burn & No. of Cases & Percentage $(\%)$ \\
Upto 40 & 11 & 08.46 \\
41 to 60 & 22 & 16.92 \\
61 to 80 & 44 & 33.84 \\
81 to 100 & 53 & 40.78 \\
Total & 130 & 100
\end{tabular}

Table 4: Predominant factors as to the cause of death

\begin{tabular}{lcc}
\hline Cause of death & No. of Cases & Percentage $(\%)$ \\
Shock & 27 & 20.77 \\
Toxemia & 30 & 23.08 \\
Septicemia & 34 & 26.15 \\
Bronchopneumonia & 11 & 08.46 \\
Pneumonia & 09 & 06.92 \\
Renal Failure & 14 & 10.77 \\
Toxic myocarditis & 05 & 03.85 \\
Total & 130 & 100
\end{tabular}

Table 5: Histopathological changes in relation to percentage (\%) of burn injury are as below

\begin{tabular}{lccccccc}
\hline Group & $\begin{array}{c}\text { Total No. of } \\
\text { Cases }\end{array}$ & Congestion & Oedema & Pneumonia & $\begin{array}{c}\text { Broncho } \\
\text {-Pneumonia }\end{array}$ & $\begin{array}{c}\text { Haemorrhage } \\
\text { Desquamative } \\
\text { Interstitial } \\
\text { Pneumonia } \\
21 \text { to } 40\end{array}$ \\
11 & $0872.72 \%$ & $0436.36 \%$ & $0218.18 \%$ & $0109.09 \%$ & - & $0327.27 \%$ \\
40 to 60 & 22 & $1463.63 \%$ & $1150.00 \%$ & $0209.09 \%$ & $0627.27 \%$ & $0104.54 \%$ & $0627.27 \%$ \\
61 to 80 & 44 & $3272.72 \%$ & $2025.45 \%$ & $0511.36 \%$ & $0409.09 \%$ & $0102.27 \%$ & $1125.00 \%$ \\
81 to 100 & 53 & $4177.35 \%$ & $2037.73 \%$ & - & - & $0101.88 \%$ & $1018.80 \%$ \\
Total & 130 & $9573.08 \%$ & $5542.31 \%$ & $0906.92 \%$ & $1108.46 \%$ & $0302.31 \%$ & $3023.08 \%$ \\
\hline
\end{tabular}

Table 6: Histopathological changes in relation to cause of death in burn injury are as below

\begin{tabular}{|c|c|c|c|c|c|c|c|}
\hline $\begin{array}{l}\text { Cause of } \\
\text { death }\end{array}$ & $\begin{array}{l}\text { Total No. of } \\
\text { Cases }\end{array}$ & Congestion & Oedema & Pneumonia & $\begin{array}{l}\text { Broncho- } \\
\text { Pneumonia }\end{array}$ & Haemorrhage & $\begin{array}{c}\text { Desquamative } \\
\text { Interstitial } \\
\text { Pneumonia }\end{array}$ \\
\hline Shock & 27 & $27100 \%$ & $0622.22 \%$ & - & - & - & - \\
\hline Toxemia & 30 & $2996.66 \%$ & $1550.00 \%$ & - & - & - & $0723.33 \%$ \\
\hline Septicaemia & 34 & $2573.52 \%$ & $2789.41 \%$ & - & - & $0102.94 \%$ & $0823.52 \%$ \\
\hline $\begin{array}{l}\text { Broncho } \\
\text { Pneumonia }\end{array}$ & 11 & $0436.36 \%$ & $0436.36 \%$ & - & $11100.00 \%$ & $0109.09 \%$ & $0763.63 \%$ \\
\hline Pneumonia & 09 & $0666.66 \%$ & - & $09100.00 \%$ & - & $0111.11 \%$ & $0777.77 \%$ \\
\hline Renal failure & 14 & $0214.28 \%$ & $0214.28 \%$ & - & - & - & $0107.14 \%$ \\
\hline $\begin{array}{l}\text { Toxic } \\
\text { myocarditis }\end{array}$ & 05 & $0240.00 \%$ & $0120.00 \%$ & - & - & - & - \\
\hline Total & 130 & $9573.08 \%$ & $5542.31 \%$ & $0906.92 \%$ & $1108.46 \%$ & $0302.31 \%$ & $3023.08 \%$ \\
\hline
\end{tabular}


Table 7: Histopathological changes in relation to survival period in burn injury are as below

\begin{tabular}{|c|c|c|c|c|c|c|c|}
\hline $\begin{array}{l}\text { Survival } \\
\text { period in } \\
\text { days }\end{array}$ & $\begin{array}{l}\text { Total No. of } \\
\text { Cases }\end{array}$ & Congestion & Oedema & Pneumonia & $\begin{array}{l}\text { Broncho- } \\
\text { Pneumonia }\end{array}$ & Haemorrhage & $\begin{array}{c}\text { Desquamative } \\
\text { Interstitial } \\
\text { Pneumonia }\end{array}$ \\
\hline Up to 01 & 20 & $20100.00 \%$ & $0525 \%$ & - & - & - & - \\
\hline 01 to 03 & 34 & $1779.41 \%$ & $1338.23 \%$ & - & $0102.94 \%$ & - & $0823.52 \%$ \\
\hline 03 to 07 & 36 & $2672.72 \%$ & $2055.55 \%$ & $0308.33 \%$ & $0205.55 \%$ & $0308.33 \%$ & $0925.00 \%$ \\
\hline 07 to 10 & 13 & $0861.53 \%$ & $0538.46 \%$ & $0215.38 \%$ & $0215.38 \%$ & - & $13100.00 \%$ \\
\hline $\begin{array}{l}10 \text { and } \\
\text { above }\end{array}$ & 27 & $1451.85 \%$ & $1244.44 \%$ & $0414.81 \%$ & $0622.22 \%$ & - & $1037.03 \%$ \\
\hline Total & 130 & $9573.08 \%$ & $5542.31 \%$ & $0906.92 \%$ & $1108.46 \%$ & - & $3023.80 \%$ \\
\hline
\end{tabular}

Table 8: Histopathological changes in brain in relation to cause of death

\begin{tabular}{lcccc}
\hline Group & $\begin{array}{c}\text { Total No of } \\
\text { cases }\end{array}$ & Congestion & Degeneration & Oedema \\
Shock & 27 & $26(96.29 \%)$ & $02(07.40 \%)$ & $01(03.70 \%)$ \\
Toxemia & 30 & $27(90.00 \%)$ & $02(06.66 \%)$ & $04(13.32 \%)$ \\
Septicaemia & 34 & $27(19.41 \%)$ & $03(08.82 \%)$ & $08(23.52 \%)$ \\
Bronchopneumonia & 11 & $03(27.27 \%)$ & - & $01(09.09 \%)$ \\
Pneumonia & 09 & $06(66.66 \%)$ & - & - \\
Renal Failure & 14 & - & - & - \\
Toxic myocarditis & 05 & $01(20.00 \%)$ & - & $14(10.76 \%)$ \\
Total & 130 & $90(69.23 \%)$ & $07(05.38 \%)$ & 14 \\
\hline
\end{tabular}

Table 9: Histopathological changes in brain in relation to survival period

\begin{tabular}{lcccc}
\hline $\begin{array}{l}\text { Survival period in } \\
\text { days }\end{array}$ & Total No. of Cases & Congestion & Degeneration & Oedema \\
Up to 01 & 20 & $20(100.00 \%)$ & - & $01(05.00 \%)$ \\
01 to 03 & 34 & $28(82.35 \%)$ & $03(08.82 \%)$ & $03(08.82 \%)$ \\
03 to 07 & 36 & $21(58.33 \%)$ & $02(05.55 \%)$ & $04(11.11 \%)$ \\
07 to 10 & 13 & $09(69.23 \%)$ & - & $01(07.69 \%)$ \\
10 and above & 27 & $12(44.44 \%)$ & $02(07.40 \%)$ & $05(18.51 \%)$ \\
Total & 130 & $90(69.23 \%)$ & $07(05.38 \%)$ & $14(10.76 \%)$ \\
\hline
\end{tabular}

Table 10: Histopathological changes in liver in relation to cause of death in burn injury are as below

\begin{tabular}{|c|c|c|c|c|c|c|c|c|}
\hline $\begin{array}{l}\text { Cause of } \\
\text { death }\end{array}$ & $\begin{array}{l}\text { Total } \\
\text { No.of } \\
\text { Cases }\end{array}$ & Congestion & $\begin{array}{c}\text { Degene- } \\
\text { ration }\end{array}$ & Necrosis & $\begin{array}{c}\text { Regene- } \\
\text { ration }\end{array}$ & $\begin{array}{c}\text { Haemo- } \\
\text { rrhage }\end{array}$ & $\begin{array}{l}\text { Hepatic } \\
\text { Sinusoidal } \\
\text { Dilatation }\end{array}$ & $\begin{array}{l}\text { Infective } \\
\text { foci }\end{array}$ \\
\hline Shock & 27 & $2177.77 \%$ & $0311.11 \%$ & $0518.51 \%$ & - & $0103.07 \%$ & - & - \\
\hline Toxemia & 30 & $1963.33 \%$ & $1136.66 \%$ & $1446.66 \%$ & $0206.66 \%$ & - & $0413.32 \%$ & $0102.94 \%$ \\
\hline Septicaemia & 34 & $1441.17 \%$ & $0823.52 \%$ & $3088.23 \%$ & $0411.76 \%$ & $0102.94 \%$ & $0823.52 \%$ & $0109.09 \%$ \\
\hline $\begin{array}{l}\text { Broncho } \\
\text { Pneumonia }\end{array}$ & 11 & $0327.27 \%$ & $0109.09 \%$ & $0109.09 \%$ & - & - & $0218.18 \%$ & - \\
\hline Pneumonia & 09 & $0555.55 \%$ & - & $0111.11 \%$ & - & - & $0111.11 \%$ & - \\
\hline $\begin{array}{l}\text { Renal } \\
\text { failure }\end{array}$ & 14 & $0214.28 \%$ & $0214.28 \%$ & - & - & - & - & - \\
\hline $\begin{array}{l}\text { Toxic } \\
\text { myocarditis }\end{array}$ & 05 & $0240.00 \%$ & - & - & - & - & - & - \\
\hline Total & 130 & $6650.76 \%$ & $2519.23 \%$ & $5139.23 \%$ & $0604.60 \%$ & $0201.52 \%$ & $1612.30 \%$ & $0201.53 \%$ \\
\hline
\end{tabular}


Table 11: Histopathological changes in liver in relation to survival period in burn injury are as below

\begin{tabular}{|c|c|c|c|c|c|c|c|c|}
\hline $\begin{array}{l}\text { Survival } \\
\text { period in } \\
\text { days }\end{array}$ & $\begin{array}{l}\text { Total No. } \\
\text { of Cases }\end{array}$ & Congestion & $\begin{array}{l}\text { Degene } \\
\text {-ration }\end{array}$ & Necrosis & $\begin{array}{c}\text { Regene- } \\
\text { ration }\end{array}$ & $\begin{array}{c}\text { Haemo- } \\
\text { rrhage }\end{array}$ & $\begin{array}{c}\text { Hepatic } \\
\text { Sinusoidal } \\
\text { Dilatation }\end{array}$ & $\begin{array}{l}\text { Infective } \\
\text { foci }\end{array}$ \\
\hline Up to 01 & 20 & $\begin{array}{c}20 \\
100.00 \%\end{array}$ & $0315.00 \%$ & $0105.00 \%$ & - & - & $0105.00 \%$ & - \\
\hline 01 to 03 & 34 & $1750.00 \%$ & $1029.41 \%$ & $1544.11 \%$ & - & - & $0205.88 \%$ & - \\
\hline 03 to 07 & 36 & $1336.11 \%$ & $0925.11 \%$ & $1747.22 \%$ & $0102.77 \%$ & $0102.77 \%$ & $0616.66 \%$ & $0202.55 \%$ \\
\hline 07 to 10 & 13 & $0323.07 \%$ & - & $0646.14 \%$ & $0107.69 \%$ & - & $0107.69 \%$ & - \\
\hline $\begin{array}{l}10 \text { and } \\
\text { above }\end{array}$ & 27 & $1344.18 \%$ & $0311.11 \%$ & $1244.44 \%$ & $0414.80 \%$ & - & $0622.22 \%$ & - \\
\hline Total & 130 & $6650.76 \%$ & $2519.23 \%$ & $5139.23 \%$ & $0604.70 \%$ & $0100.76 \%$ & $1612.30 \%$ & $0201.53 \%$ \\
\hline
\end{tabular}

Table 12: Histopathological changes in spleen in relation to cause of death in burn injury are as below

\begin{tabular}{|c|c|c|c|c|c|c|c|c|}
\hline $\begin{array}{l}\text { Cause of } \\
\text { death }\end{array}$ & $\begin{array}{l}\text { Total } \\
\text { No.of } \\
\text { Cases }\end{array}$ & Congestion & $\begin{array}{c}\text { Degene- } \\
\text { ration }\end{array}$ & Necrosis & $\begin{array}{c}\text { Follicular } \\
\text { Hyper- } \\
\text { plasia }\end{array}$ & $\begin{array}{c}\text { Hyline like } \\
\text { material in } \\
\text { arteriole }\end{array}$ & $\begin{array}{c}\text { Haemo- } \\
\text { rrhage }\end{array}$ & $\begin{array}{l}\text { Acute } \\
\text { Inflam. }\end{array}$ \\
\hline Shock & 27 & $\begin{array}{c}27 \\
100.00 \%\end{array}$ & $0311.11 \%$ & - & - & - & - & - \\
\hline Toxemia & 30 & $2893.33 \%$ & $0516.66 \%$ & $0666.00 \%$ & - & - & - & - \\
\hline Septicaemia & 34 & $\begin{array}{c}34 \\
100.00 \%\end{array}$ & $0617.66 \%$ & $0617.66 \%$ & $0102.94 \%$ & $0514.70 \%$ & $0205.88 \%$ & $0102.94 \%$ \\
\hline $\begin{array}{l}\text { Broncho } \\
\text { Pneumonia }\end{array}$ & 11 & $0545.45 \%$ & $0109.90 \%$ & - & - & - & - & - \\
\hline Pneumonia & 09 & $0555.55 \%$ & $0111.11 \%$ & - & - & - & - & - \\
\hline Renal failure & 14 & $0321.42 \%$ & $0107.14 \%$ & $0107.14 \%$ & - & - & - & - \\
\hline $\begin{array}{l}\text { Toxic } \\
\text { myocarditis }\end{array}$ & 05 & $0240.00 \%$ & - & $0120.00 \%$ & - & - & - & - \\
\hline Total & 130 & $\begin{array}{c}104 \\
80.00 \%\end{array}$ & $1713.07 \%$ & $1006.69 \%$ & $0100.76 \%$ & $0503.84 \%$ & $0201.53 \%$ & $0100.76 \%$ \\
\hline
\end{tabular}

Table 13: Histopathological changes in spleen in relation to survival period in burn injury are as below

\begin{tabular}{|c|c|c|c|c|c|c|c|c|}
\hline $\begin{array}{l}\text { Survival } \\
\text { period in } \\
\text { days }\end{array}$ & $\begin{array}{l}\text { Total No. } \\
\text { of Cases }\end{array}$ & Congestion & $\begin{array}{l}\text { Degene- } \\
\text { ration }\end{array}$ & Necrosis & $\begin{array}{c}\text { Follicular } \\
\text { Hyper } \\
\text { plasia }\end{array}$ & $\begin{array}{c}\text { Hyline like } \\
\text { material in } \\
\text { arteriole }\end{array}$ & $\begin{array}{c}\text { Haemo- } \\
\text { rrhage }\end{array}$ & $\begin{array}{r}\text { Acute } \\
\text { Inflam. }\end{array}$ \\
\hline Up to 01 & 20 & $\begin{array}{c}20 \\
100.00 \%\end{array}$ & $0210.00 \%$ & - & - & - & - & - \\
\hline 01 to 03 & 34 & $\begin{array}{c}34 \\
100.00 \%\end{array}$ & $1764.00 \%$ & $0102.94 \%$ & - & $0102.94 \%$ & $0102.94 \%$ & - \\
\hline 03 to 07 & 36 & $2775.00 \%$ & $0411.11 \%$ & $0411.11 \%$ & $0102.77 \%$ & $0102.77 \%$ & $0102.77 \%$ & - \\
\hline 07 to 10 & 13 & $0969.73 \%$ & - & $0107.69 \%$ & - & $0107.69 \%$ & $0107.69 \%$ & $0107.69 \%$ \\
\hline $\begin{array}{l}10 \text { and } \\
\text { above }\end{array}$ & 27 & $1451.85 \%$ & $0518.51 \%$ & $0414.81 \%$ & - & $0311.11 \%$ & - & - \\
\hline Total & 130 & $\begin{array}{c}104 \\
80.00 \%\end{array}$ & $1713.07 \%$ & $1006.69 \%$ & $0100.76 \%$ & $0604.61 \%$ & $0302.30 \%$ & $0100.76 \%$ \\
\hline
\end{tabular}

changes in brain on $2^{\text {nd }}$ day onwards in few cases. ${ }^{8}$

Congestion of heart was seen to $90 \%$ cases which died within first 24 hours of sustaining burns. It was also predominant finding in few cases which survived beyond 24 Hrs. Focal minimal interstitial myocarditis was observed in $5(3.84 \%)$ cases. This could be due to toxins produced. Clark has stated metabolic toxin as a cause of myocarditis in $6 \%$ cases of burns. Teplitz mentioned that, the heart in burns showed no specific or significant primary cardiac lesion. He observed interstitial myocarditis in $08.80 \%$ cases. Ahauer et al stated burn toxin which caused chemical injury to myocardium, as a cause of myocarditis.

Congestion of lung was seen in all cases which died within 24 hours of sustaining burns. Foley stated that congestion was frequently the only finding in patients with extensive who died in early post burn period.

In the present study oedema was found in $42.30 \%$ cases which is also comparable to those of other studies (Shook, 1969 (66\%) Foley, 1969(30.7\%) Teplitz, 1969 (32\%) Stone et al $1969(40.50 \%)$ and Pruitt et al (1970) 30.80\%. Teplitz 
Table 14: Histopathological changes in kidney in relation to cause of death in burn injury are as below

\begin{tabular}{|c|c|c|c|c|c|c|c|}
\hline $\begin{array}{l}\text { Cause of } \\
\text { death }\end{array}$ & $\begin{array}{c}\text { Total } \\
\text { Number of } \\
\text { Cases }\end{array}$ & Congestion & $\begin{array}{c}\text { Degene- } \\
\text { ration }\end{array}$ & Necrosis & $\begin{array}{c}\text { Casts RBC } \\
\text { in tubule }\end{array}$ & Haemo- rrhage & $\begin{array}{c}\text { Interstitial } \\
\text { Nephritis }\end{array}$ \\
\hline Shock & 27 & $2696.29 \%$ & $0207.40 \%$ & $0518.51 \%$ & - & - & - \\
\hline Toxemia & 30 & $2066.66 \%$ & $0723.33 \%$ & $1136.66 \%$ & $0206.66 \%$ & $0206.66 \%$ & - \\
\hline Septicaemia & 34 & $1750.00 \%$ & $0926.47 \%$ & $2367.64 \%$ & $0102.94 \%$ & $0205.89 \%$ & - \\
\hline $\begin{array}{l}\text { Broncho- } \\
\text { Pneumonia }\end{array}$ & 11 & $0218.18 \%$ & $0436.36 \%$ & - & - & - & - \\
\hline Pneumonia & 09 & $0555.55 \%$ & $0111.11 \%$ & $0111.11 \%$ & - & - & - \\
\hline Renal failure & 14 & $0107.14 \%$ & - & $0321.42 \%$ & - & - & $0214.28 \%$ \\
\hline $\begin{array}{l}\text { Toxic } \\
\text { myocarditis }\end{array}$ & 05 & $0240.00 \%$ & - & - & - & - & - \\
\hline Total & 130 & $7356.15 \%$ & $2317.69 \%$ & $4333.07 \%$ & $0302.30 \%$ & $0403.07 \%$ & $0201.53 \%$ \\
\hline
\end{tabular}

Table 15: Histopathological changes in kidney in relation to survival period in burn injury are as below

\begin{tabular}{lccccccc}
\hline $\begin{array}{l}\text { Survival } \\
\text { period in } \\
\text { days }\end{array}$ & $\begin{array}{c}\text { Total } \\
\text { Number of } \\
\text { Cases }\end{array}$ & Congestion & Degeneration & Necrosis & $\begin{array}{c}\text { Casts RBC } \\
\text { in tubule }\end{array}$ & $\begin{array}{c}\text { Haemo- } \\
\text { rrhage }\end{array}$ & $\begin{array}{c}\text { Interstitial } \\
\text { Nephritis }\end{array}$ \\
Up to 01 & 20 & $20100.00 \%$ & $0105.00 \%$ & $0420.00 \%$ & - & - & - \\
01 to 03 & 34 & $2676.47 \%$ & $1029.41 \%$ & $0823.52 \%$ & $0205.88 \%$ & $0205.88 \%$ & - \\
03 to 07 & 36 & $1747.22 \%$ & $0308.33 \%$ & $1438.88 \%$ & $0102.77 \%$ & $0205.55 \%$ & - \\
07 to 10 & 13 & $0430.76 \%$ & $0107.69 \%$ & $0646.15 \%$ & - & - & - \\
10 and above & 27 & $0622.22 \%$ & $0829.62 \%$ & $1140.74 \%$ & - & $07.40 \%$ \\
Total & 130 & $7356.15 \%$ & $2317.69 \%$ & $4333.07 \%$ & $0302.30 \%$ & $0403.07 \%$ & $0201.53 \%$ \\
\hline
\end{tabular}

Table 16: Histopathological changes in adrenal in relation to cause of death in burn injury are as below

\begin{tabular}{lccccccc}
\hline $\begin{array}{l}\text { Cause of } \\
\text { death }\end{array}$ & $\begin{array}{c}\text { Total } \\
\text { Number of } \\
\text { Cases }\end{array}$ & Congestion & Degeneration & Necrosis & $\begin{array}{c}\text { Haemo- } \\
\text { rrhage }\end{array}$ & Fat depletion & Infective foci \\
Shock & 27 & $2281.48 \%$ & $0311.11 \%$ & $0933.33 \%$ & $0222.22 \%$ & $0518.51 \%$ & - \\
Toxemia & 30 & $1963.33 \%$ & $0723.33 \%$ & $1446.66 \%$ & $0310.00 \%$ & $0413.33 \%$ & $0103.33 \%$ \\
Septicaemia & 34 & $2161.76 \%$ & $1338.23 \%$ & $2161.76 \%$ & $0720.58 \%$ & $0926.47 \%$ & $0102.94 \%$ \\
Broncho & 11 & $0436.36 \%$ & $0218.18 \%$ & $0109.09 \%$ & $0109.09 \%$ & $0218.18 \%$ & - \\
$\begin{array}{l}\text { Pneumonia } \\
\text { Pneumonia }\end{array}$ & 09 & $0444.44 \%$ & $0111.11 \%$ & $0222.22 \%$ & $0111.11 \%$ & - & - \\
$\begin{array}{l}\text { Renal failure } \\
\text { Toxic }\end{array}$ & 14 & $0321.42 \%$ & $0107.14 \%$ & - & - & - & - \\
myocarditis & 05 & $0240.00 \%$ & - & - & - & $2015.38 \%$ & $0201.53 \%$ \\
Total & 130 & $7557.69 \%$ & $2720.76 \%$ & $4736.15 \%$ & $1410.76 \%$ & 20 \\
\hline
\end{tabular}

Table 17: Histopathological changes in adrenal in relation to survival period in burn injury are as below

\begin{tabular}{lccccccc}
\hline $\begin{array}{l}\text { Survival } \\
\text { period in } \\
\text { days }\end{array}$ & $\begin{array}{c}\text { Total } \\
\text { Number of } \\
\text { Cases }\end{array}$ & Congestion & Degeneration & Necrosis & $\begin{array}{c}\text { Haemo- } \\
\text { rrhage }\end{array}$ & $\begin{array}{c}\text { Fat } \\
\text { depletion }\end{array}$ & Infective foci \\
$\begin{array}{l}\text { Up to } 01 \\
01 \text { to } 03\end{array}$ & 20 & $20100.00 \%$ & $0105.00 \%$ & $0630.00 \%$ & - & $0525.00 \%$ & - \\
03 to 07 & 34 & $2058.02 \%$ & $0926.47 \%$ & $1338.23 \%$ & $0308.82 \%$ & $0205.88 \%$ & $0205.88 \%$ \\
07 to 10 & 36 & $2158.33 \%$ & $0513.88 \%$ & $1233.33 \%$ & $0822.22 \%$ & $0719.44 \%$ & - \\
10 and & 13 & $0430.76 \%$ & - & $0753.84 \%$ & - & - & - \\
above & 27 & $1037.03 \%$ & $1244.44 \%$ & $0933.33 \%$ & $0311.11 \%$ & $0622.22 \%$ & $0201.53 \%$ \\
Total & 130 & $7557.69 \%$ & $2720.76 \%$ & $4736.15 \%$ & $1410.76 \%$ & $2015.38 \%$ & 0 \\
\hline
\end{tabular}


thought congestion and oedema as reflection of systemic circulatory failure rather than primary pulmonary insult. Foley (1969) considered it as an effect of acute inhalation injury. He strongly suggested that inhalation of toxic gases rather than thermal injury, was responsible for oedema. He blamed congestion and oedema as a cause of death.

In this study changes in liver were found as congestion $66(50.76 \%)$ cases. Similar finding were noted by Artz et al (1979) was found in 50\% cases. Jackson et al (1963) and Panke et al (1985) also quoted congestion and necrosis in liver in their studies.

The hepatic necrosis was found in $49.11 \%$ cases who survived up-to 3days. Similar findings were noted by Wilson (1939), Teplitz (1939) as midzonal necrosis. These were also noted by Zink (1940). Regeneration was found in $04.60 \%$ cases in this study. This histological finding is consistent with appearance of large nuclei, with prominent nuclear membrane, bi-nucleation of hepatic cells with mitosis at places. ${ }^{8-10}$

The predominant findings in spleen were congestion in $104(80 \%)$ cases followed by degeneration $17(13.07 \%)$, necrosis $10(06.69 \%)$, hyaline like material in arteriolar wall in $5(03.84 \%)$, haemorrhage in $3(02.30 \%)$ follicular hyperplasia and acute inflammation in 1 case. ${ }^{11}$

Predominant findings in kidneys were congestion in 73 (56.15\%), followed by necrosis $43(33.07 \%)$ degeneration $23(17.69 \%)$ haemorrhage $4(03.07 \%)$ RBC casts in tubule in $3(02.30 \%)$ and interstitial nephritis $2(01.53 \%)$ cases. Renal cortical necrosis was found in $33.07 \%$ cases. $^{12}$

Congestion of adrenal gland $75(57.69 \%)$ and was followed by necrosis in $47(36.15 \%)$ and degeneration in $29(20.76 \%)$ cases. Fat depletion was found in $20(15.38 \%)$ cases. $^{13}$

In this study almost all vital and major organs were included for histopathological examination. Though the number was sizeable, the study was dependent on available cases.

Simultaneously study from histo-chemistry point of view could have been carried out.

\section{Summary \& Conclusion}

A prospective autopsy study is carried out in 130 cases of deaths due to burns from February 1988 to November 1989. Out of 130 cases 35 were males and 95 were females. $80.76 \%$ subject belonged to the Hindu religion. The manner of sustaining burns by bursting of stove was predominant etiological factor. Use of kerosene as accelerant was very common $(69.23 \%)$. Based on history given by the patients, accidental burns were the most common, followed by suicidal and homicidal. The age group 21 to 40 years was involved in $59.24 \%$ cases. Most of the cases died within 7 days of sustaining burns.

91.54\% cases sustained more than $40 \%$ Burns. Microscopic observations of visceral organs: brain, heart, lung, liver, spleen, kidney and adrenal were recorded and analyzed keeping in view the time sequence after burn incidence. Congestion was seen in all cases which died within first 24 hours. On $2^{\text {nd }}$ day onwards in brain, heart the degeneration and focal minimal interstitial myocarditis were observed subsequently. In lung oedema, bronchopneumonia, pneumonia and desquamative interstitial pneumonitis were important findings. In liver degenerative changes, necrosis, regeneration and hepatic sinusoidal dilatation were seen on $2^{\text {nd }}$ day onwards. Similarly spleen showed degeneration necrosis, presence of hyaline like material in arteriolar walls and haemorrhage. In kidney degenerative changes and necrosis of tubular epithelium were observed from1st day. Adrenal showed congestion, degeneration, necrosis, fat depletion and haemorrhage from $1^{\text {st }}$ day onwards. However, it is very difficult to pin-point a cause of death, particularly a single one. It appears that combination of factors like neurogenic shock, hypovolemic shock, toxemia, infections and respiratory complications including presence of bronchopneumonia, pneumonia etc. along with pulmonary oedema, are responsible. Cerebral oedema, pulmonary oedema and generalized congestion also seem to be important in adversely affecting the life processes. In few cases myocarditis with degenerative changes may also contribute to death. In certain number of cases liver necrosis may play an important role by causing metabolic derangement in ultimate fatal result. Acute renal tubular necrosis with varying degree of degenerative changes in kindly also appear to be important contributory factors in causing death particularly in 3 to 7 days. Contrary to observations by many workers in earlier studies, present study shows significant and extensive changes in adrenal in form of congestion, fat depletion, haemorrhage and necrosis in various combinations.

\section{Source of Funding}

None.

\section{Conflict of Interest}

None.

\section{References}

1. Olivera-Martinez I, Viallet JP, Michon F, Pearton DJ, Dhouailly D. The different steps of skin formation in vertebrates. Int J Dev Biol. 2004;48(2-3):107-15.

2. Abrol A, Saraf R, Singh S. Thermal and electrical burns in Jammu Province. J K Sci. 2005;7:61-3.

3. Artz CP, Mocrief JA, Pruitt BA. Burns- A Team approach. London: W.B. Saunders Co; 1979.

4. Sharma BR, Harish D, Sharma V, Vij K. Kitchen accidents vis-a-vis dowry deaths. Burns. 2002;28(3):250-3.

5. Forester DW, Richaerdson WIR. Causes of burn in Oklahoma. J Okla Med Assoc. 1959;52:713-4.

6. Parks JG, Noguchi TT, Klatt EC. The Epidemiology of Fatal Burn Injuries. J Forensic Sci. 1989;34(2):12650J. 
7. Bull JP. Causes, Prognosis and prevention of burns. Med Press. 1958;239:205-6.

8. Erb IH, Morgan EM, Farmar AW. The pathology of burns: the pathological picture as revealed at autopsy in a series of 61 fatal cases treated at the hospital for sick children, Toronto, Canada. Ann Surg. 1943; 117:234-55.

9. Taylor FW, Gumbert JL. Cause of death from the burns: role of respiratory damage. Ann Surg. 1965;161(4):497-501.

10. Pathology RV. Mortality and Prognosis of Burns: A Review of 54 Critical and Fatal Cases. Can Med Assoc J. 1967;97(9):445-9.

11. Wilson WC, Macgregor AR, Stewart CP. The clinical course and pathology of burns and scalds under modern methods of treatment. Br J Surg. 1938;25(100):826-65.

12. Shinde AB, Keoliya AN. Study of Burn Deaths with Special Reference to histopathology in India. Ind Jour of Basic \& Applied Medical Research. 2013;2(8):1153-9.
13. Mzezewa S, Jonsson K, Aberg M, Salemark L. A prospective study on the epidemiology of burns in patients admitted to the Harare burn units. Burns. 1999;25(6):499-504.

\section{Author biography}

Avinash J Pujari Assistant Professor

Rahul V Kedare Assistant Professor

Cite this article: Pujari AJ, Kedare RV. A histopathological study of visceral organs in death due to burns. Indian J Forensic Community Med 2020;7(2):84-91 